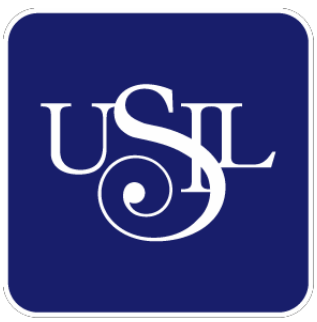

UNIVERSIDAD

SAN IGNACIO

DE LOYOLA

ESCUELA DE POSTGRADO

\title{
PLAN ESTRATÉGICO: EMPRESA DE SERVICIOS DE COURIER Y PAQUETERÍA, GTL EXPRESS S.A.C. EN LA CIUDAD DE LIMA
}

Trabajo de Investigación para optar el grado de:

EDUARDO ASTETE ROCHA

Maestro en Ciencias Empresariales con Mención en Gestión del Capital Humano

CARLOS MIGUEL LINDO MONGE

Maestro en Ciencias Empresariales con Mención en Gestión del Capital Humano

MIGUEL GABRIEL QUIROZ RIVERO

Maestro en Ciencias Empresariales con Mención en Gestión de Proyectos

Asesor:

Luis Fernando Peredo Rojas

Lima - Perú

2019 
"Plan Estratégico: Empresa de servicios de courier y paquetería GTL Express S.A.C. en la ciudad de Lima" 


\section{Dedicatoria}

Dedicamos este trabajo a nosotros mismos, hemos tenido muchos retos, siendo uno de ellos el post-grado de la USIL. Tras vernos inmersos en él, uno se plantea que más allá de ser un reto, es una base, no solo para el entendimiento del campo en el que nos hemos visto involucrados, sino para nuestras vidas y futuro.

A nuestros padres y familia por la motivación permanente hacia el logro de nuestros objetivos profesionales. 


\section{Agradecimiento}

Agradecemos a la empresa GTL Express S.A.C. por la confianza y el apoyo brindado durante todo el proceso de desarrollo de nuestro proyecto, ya que sin su ayuda no hubiera sido posible culminar el presente plan estratégico.

A nuestro asesor Dr. Luis Fernando Peredo Rojas que fue nuestro guía y siempre estuvo dispuesto ayudarnos en la mejora de nuestro proyecto. 


\section{Resumen ejecutivo}

El presente plan estratégico 2018-2022 ha sido desarrollado para la empresa GTL Express S.A.C., el cual dará los lineamientos que se requiere para mantenerse competitivo en el mercado. La empresa se encuentra comprendida en el sector transporte, almacenamiento, correo y mensajería, cuya fortaleza es el conocimiento de las leyes aduaneras, optimizando el tiempo de entrega y el servicio personalizado. GTL Express S.A.C. fue creada para atender la demanda de clientes que buscan un mejor servicio de encomiendas.

En la actualidad, existen factores macroeconómicos que benefician el desarrollo y la sostenibilidad de la empresa, entre ellos se encuentran el PBI, la inflación, el crecimiento de las importaciones, el aumento de las compras electrónicas y la disminución de la pobreza.

El plan estratégico propone integrar la estrategia de penetración de mercado en base a los siguientes criterios: a) Crear valor a los accionistas (rentabilidad de los accionistas), b) Incrementar las ventas en una tasa promedio de $14 \%$ anual, c) Crecer de manera sostenible entre los años 2018-2022, d) Fidelizar a los Clientes, e) Capacitar al personal e f) Identificar los macro procesos.

En relación a la evaluación económica financiera del presente plan, se determinó que la inversión inicial requerida ascendió a S/. 113.782 soles siendo el 43.23\% inversión fija y el $56.77 \%$ capital de trabajo. 
En lo referente a los indicadores de evaluación de inversiones, y en relación a los indicadores económicos bajo los supuestos de planeación se han obtenido los siguientes resultados: un VANE de S/. 231,797, y un TIRE de 195.75\%, que aparentemente pareciera bastante excesivo debido a la distorsión que se genera por la metodología misma del cálculo de la TIR. Este último solo mide el retorno sobre la inversión fija inicial, y no sobre el total de inversión fija y corriente, por lo que se agregó el indicador beneficio costo el cual muestra un resultado de retorno sobre el total de la inversión de solo el $10 \%$

En lo que respecta a la evaluación financiera, bajo los supuestos de planeación, se han obtenido los siguientes resultados: VANF de S/. 241,848 y un TIRF de $344.21 \%$, que aparentemente también pudiera parecer exageradamente alto al igual que TIRE, pero como ya se explicó, la deficiencia en la metodología del TIR da origen a este resultado distorsionado. Por lo tanto, debido al tiempo en que viene trabajando la empresa, el análisis TIRE es el más adecuado. 


\section{Índice de contenido}

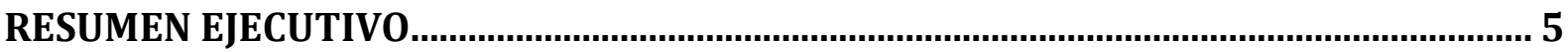

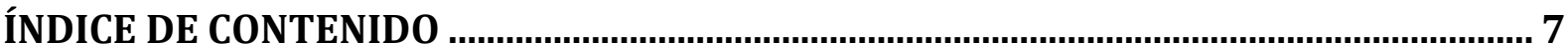

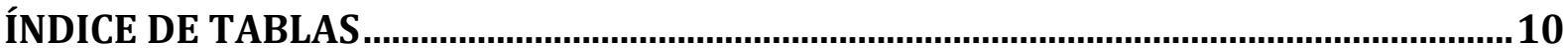

ÍNDICE DE FIGURAS ...................................................................................................11

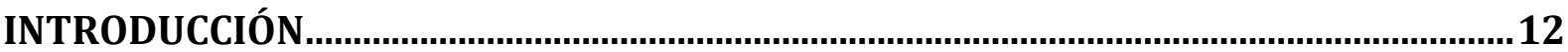

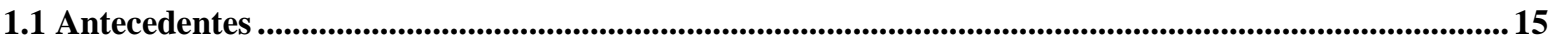

1.2 Determinación del problema u oportunidad .......................................................................................................16

1.3 Justificación del Proyecto .................................................................................................................................19

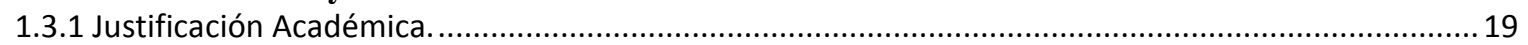

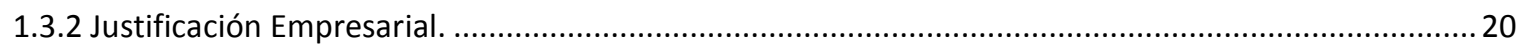

1.4 Objetivos generales y específicos....................................................................................................................... 20

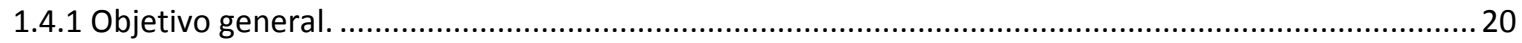

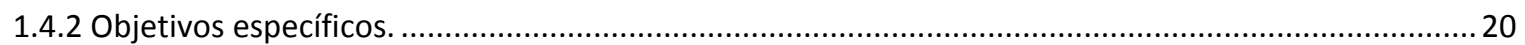

1.5 Alcances y limitaciones de la investigación ..........................................................................................................21

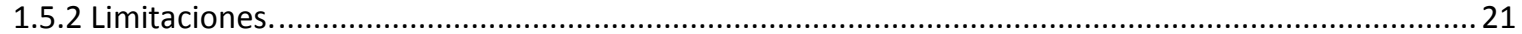

LA EMPRESA

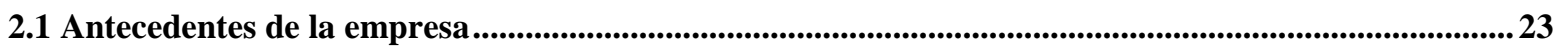

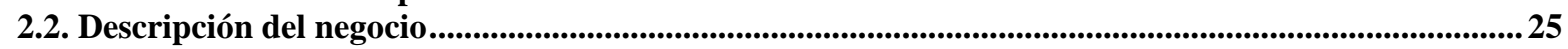

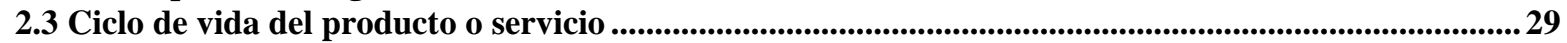

2.4 Estructura organizacional actual de la empresa .....................................................................................32

2.5 Situación del mercado y situación financiera actual de la industria.................................................35

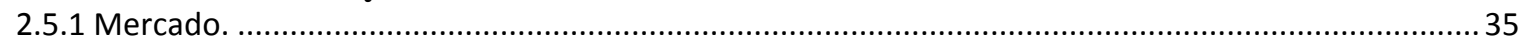

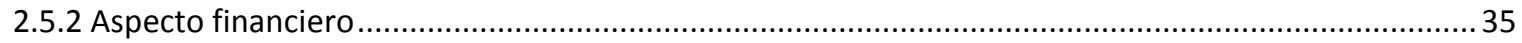

\section{CAPÍTULO III: FORMULACIÓN DE LA VISIÓN, MISIÓN Y VALORES DE LA EMPRESA41}

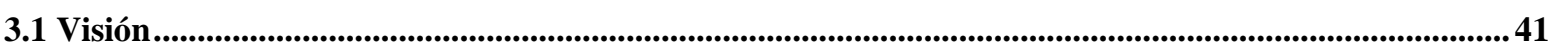

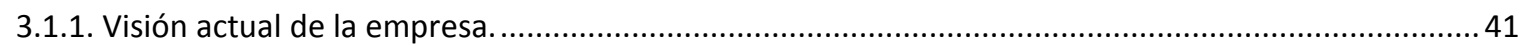

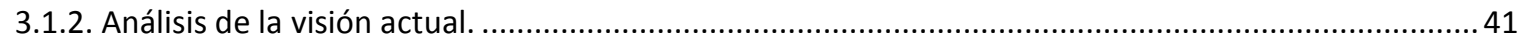

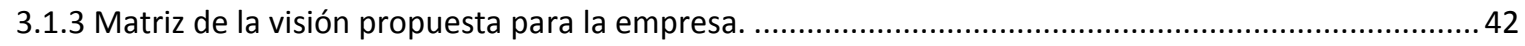

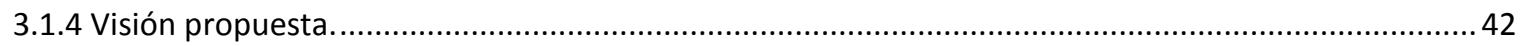




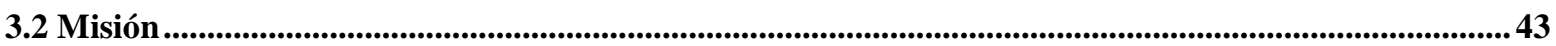

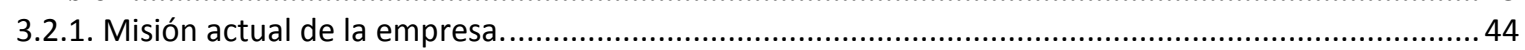

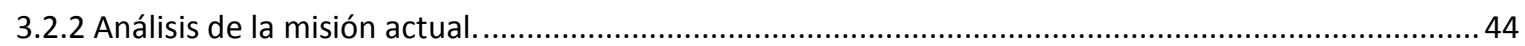

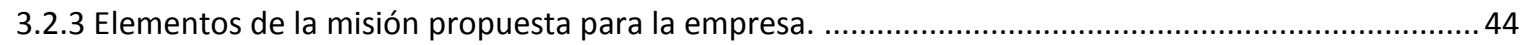

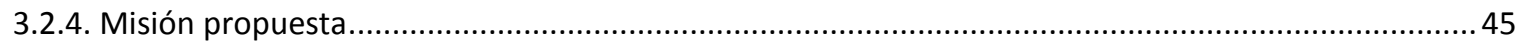

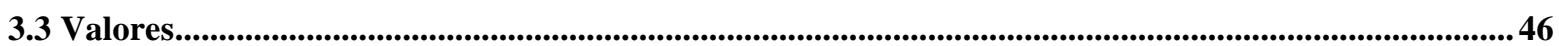

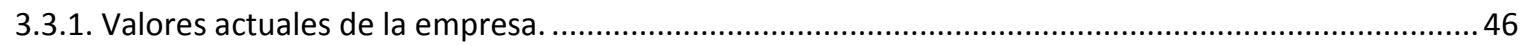

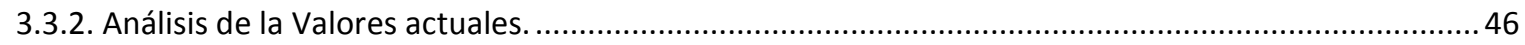

3.3.3. Elementos de los valores propuestos para la empresa. ........................................................... 46

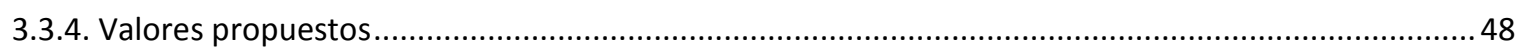

3.4 Alineamiento estratégico de la Visión, misión y valores de la empresa .............................................49

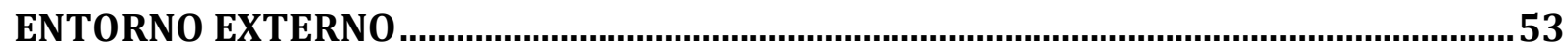

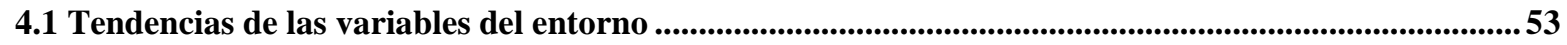

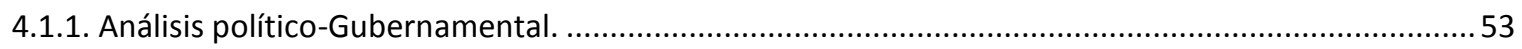

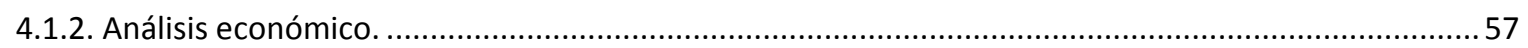

4.2 Impacto en clientes / proveedores de cada una de las variables del entorno.........................................74

4.3 Efecto en la empresa de cada una de las variables del entorno. ................................................................75

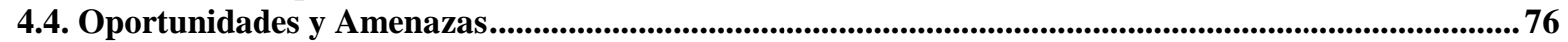

4.5 Matriz de evaluación de los factores externos EFE.................................................................................78

ANÁLISIS DE LA INDUSTRIA .............................................................................................84

5.1 Descripción del mercado (demanda) e Industria (Oferta) .....................................................................8 84

5.2 Descripción de las cinco fuerzas competitivas de la industria. ................................................................90

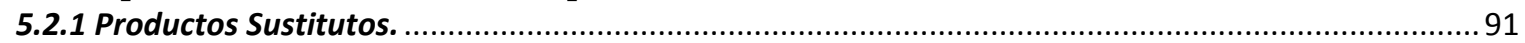

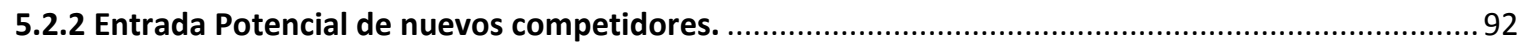

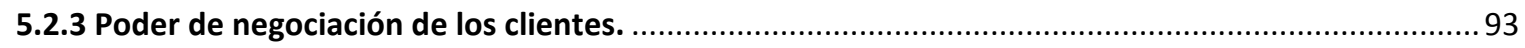

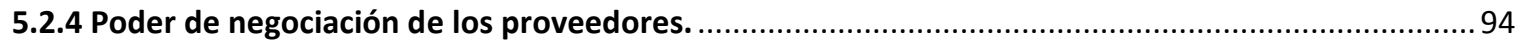

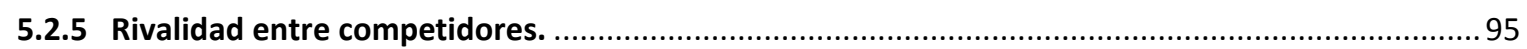

5.3 Matriz de actividad de cada una de las cinco fuerzas. .....................................................................98

5.4 Análisis del grado de atractividad de la industria ..................................................................................101

CAPÍTULO VI 
6.1 Descripción de las actividades de la cadena de valor de la empresa:................................................111

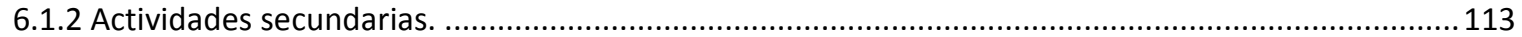

6.2 Indicadores de cada una de las actividades de la cadena de valor. ........................................................114

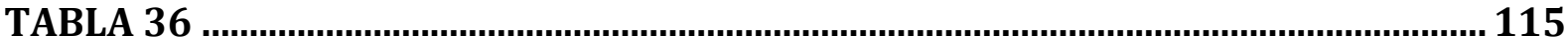

DIRECCIÓN: DETERMINACIÓN DE FORTALEZAS Y DEBILIDADES ............................ 115

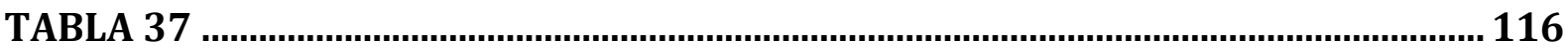

OPERACIONES: DETERMINACIÓN DE FORTALEZAS Y DEBILIDADES.......................116

6.3 Benchmarking y comparación con líderes de la industria de cada una de actividades de la cadena de

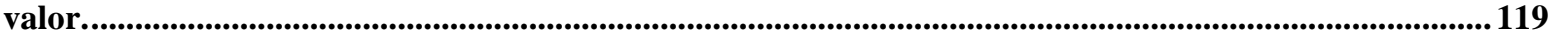

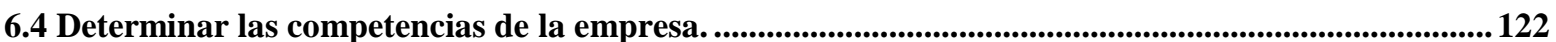

6.5 Identificación y determinación de las ventajas competitivas de la empresa. ........................................122

6.6 Matriz de evaluación de los factores internos EFI. ........................................................................124

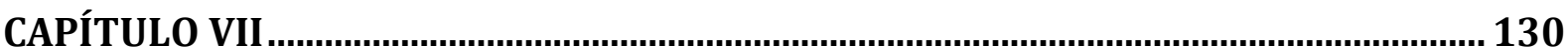

FORMULACIÓN DE OBJETIVOS Y DISEÑO DE LAS ESTRATEGIAS................................ 130

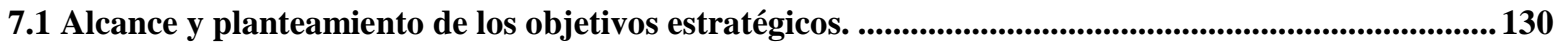

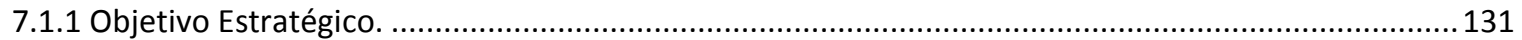

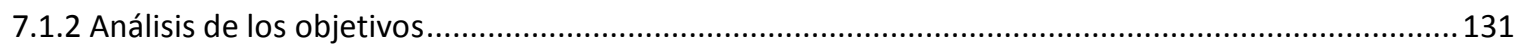

7.2.1 Matrices de formulación de estrategias............................................................................. 136

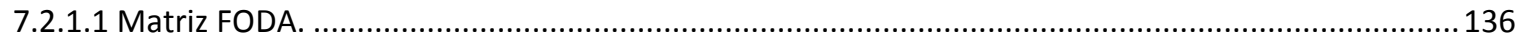

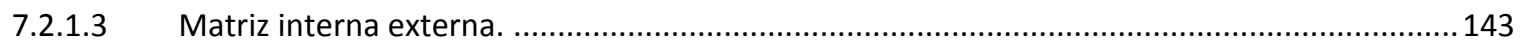

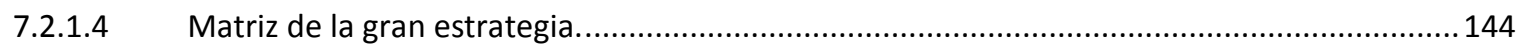

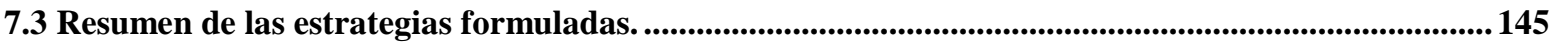

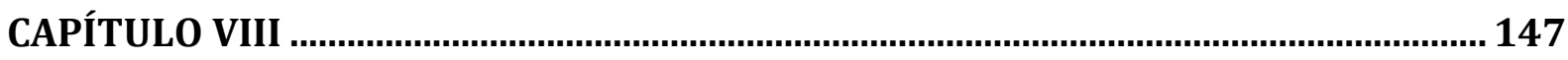

SELECCIÓN DE LA ESTRATEGIA.................................................................................. 147

8.1 Método factores estrategias claves ................................................................................................................ 147

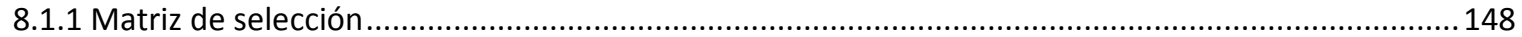

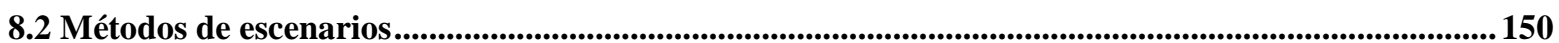

8.2.1 Descripción de escenarios considerados................................................................................... 151

8.3 Matriz de planeación estratégica cuantitativa MPEC. ..................................................................................155

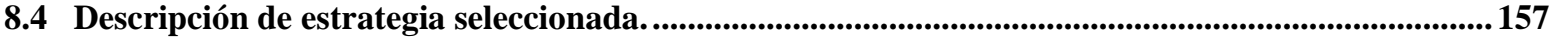

8.5 Descripción de estrategia contingente.............................................................................................157

9.2 Objetivos específicos según el mapa de la estrategia. ..................................................................163 
9.3 Indicadores para cada uno de los objetivos específicos .............................................................165

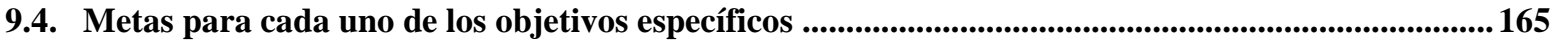

9.5 Iniciativas (acciones a llevar a cabo para cada uno de los objetivos específicos) ...............................166

9.7 Presupuesto de cada una de las iniciativas ....................................................................................167

9.8 Cronograma de cada una de las iniciativas ........................................................................................... 168

CAPÍTULO X

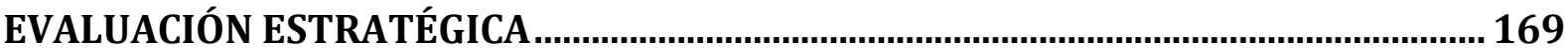

$10.1 \quad$ Evaluación Cualitativa ......................................................................................................................169

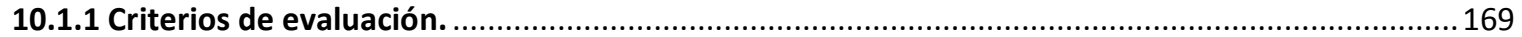

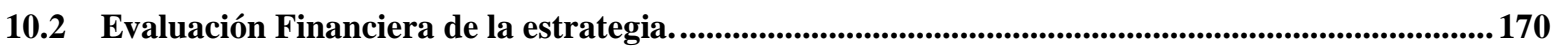

CONCLUSIONES Y RECOMENDACIONES ........................................................................ 202

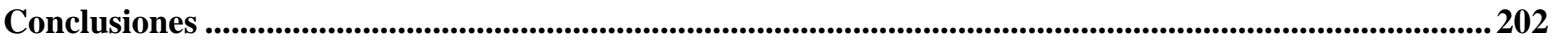

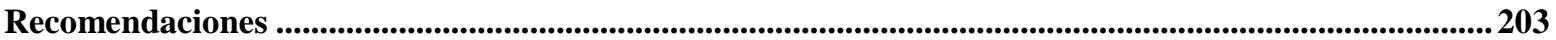

REFERENCIAS ELECTRÓNICAS ................................................................................... 231

Índice de tablas

Tabla 71. Proyección del Flujo de Caja de GTL Express Con Estrategia ............................. 185

Tabla 72. Flujo de Fondos Económicos de GTL Express ........................................................ 193

Tabla 73. Beneficio Costo Económico de GTL Express (TIRE) ............................................ 195

Tabla 74. Periodo de Recuperación de GTL Express (TIRE) ............................................... 196 
Tabla 75. Flujo de Fondos Financieros de GTL Express (TIRE)......................................... 196

Tabla 76. Beneficio Costo Económico de GTL Express (TIRF) ............................................ 198

Tabla 77. Periodo de Recuperación de GTL Express (TIRF) ............................................... 199

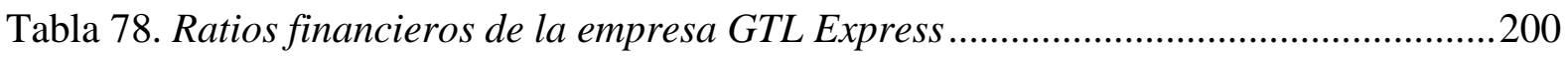

\section{Índice de figuras}

Figura 1. Producción de transporte, almacenamiento, correo y mensajería, 2010 - 2017 .......21

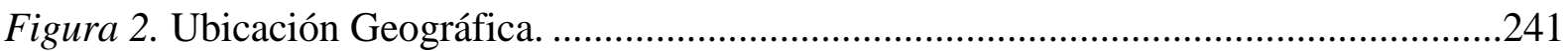

Figura 3. Diagrama de Procesos de GTL Express SAC ..........................................................29

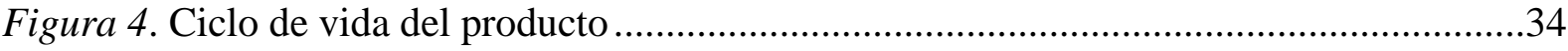




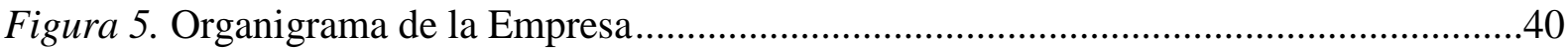

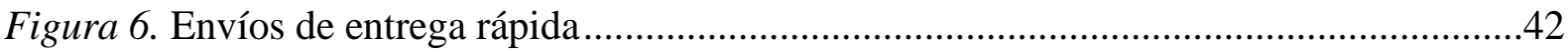

Figura 7. PBI del Perú: Nacional, Transporte y Telecomunic., Enero 2014 - Junio 2015 ......44

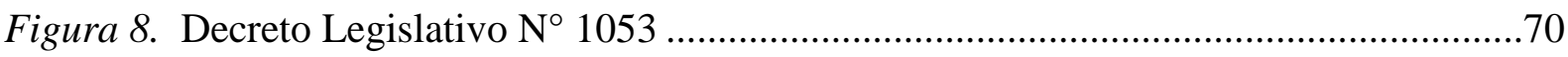

Figura 9. Tráfico postal nacional e internacional año 2016..............................................90

Figura 10. Tráfico postal internacional según tipo de envío (participación) I S 2015 .............91

Figura 11. Tráfico postal internacional de pequeños paquetes según ruta de envío I S 2015.92

Figura 12. Tráfico postal internacional según empresa (participación) I S 2015.

Figura 13. Tráfico postal internacional de pequeños paquetes según empresa (participación)

I S 2015

Figura 14. Modelo de las cinco fuerzas de Porter .905

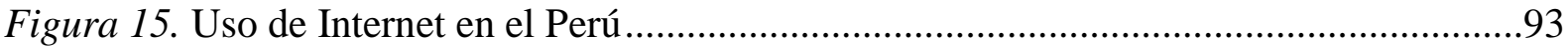

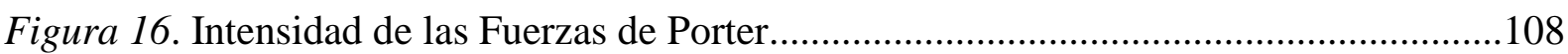

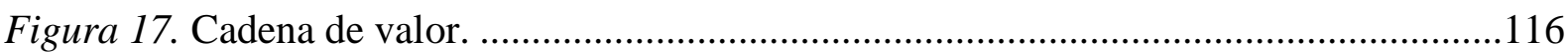

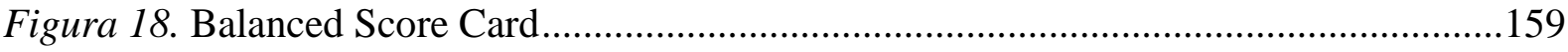

\section{Introducción}

El sector transporte, almacenamiento, correo y mensajería es uno de los sectores con mayor desarrollo y dinamismo en los últimos años, tanto en el Perú como en otras naciones del mundo. Estos efectos de crecimiento y cambios constantes obligaron a las empresas del 
sector a mantenerse más informados y competitivos, con el objetivo de ofrecer mejor calidad en los servicios y mayor satisfacción para los clientes.

Las empresas dedicadas a la investigación en el Perú mencionaron que el sector transporte, almacenamiento, correo y mensajería tienen una gran importancia en el dinamismo de la economía peruana y en la globalización del negocio, permitiendo el enlace rápido con diversos países del mundo. Esto trajo como consecuencia que cada día más personas acceden a nuevos mercados mediante el comercio electrónico, tal como se señaló en el informe In Crescendo Ciencias Contables \& Administrativas (ICC C\&A); ello facilitó que las empresas adquieran información sobre nuevos proveedores que les aseguró la adquisición de sus diferentes recursos para sus procesos de producción y comercialización (ICC C\&A, 2017).

GTL Express S.A.C. brinda el servicio de courier y paquetería en Lima Metropolitana y Callao, el cual consiste en la importación de documentos o paquetes de una determinada dimensión y peso limitado. Los envíos corresponden a productos adquiridos en el exterior por los clientes. GTL Express se encarga de la recepción, traslado, desaduanaje, nacionalización y entrega del paquete al destino indicado por el cliente.

GTL Express cuenta con un operador logístico en la ciudad de Miami, el cual se encarga de recepcionar los documentos o paquetería, consolidar y gestionar el envío hacia al Perú. Este servicio se caracteriza por tener la velocidad, seguridad, especialización y personalización en el proceso y ejecución del reparto.

El servicio de courier de GTL Express va dirigido no solo al público en general, sino también a empresas y entidades privadas que requieran de envíos de forma rápida y segura; y busquen en una empresa de courier la calidad en el servicio y efectividad en sus envíos. 
Es en este contexto donde se origina la motivación para el desarrollo del presente plan estratégico aplicando la metodología basada en el proceso estratégico de Fred David, el cual se compone de un conjunto de actividades que se desarrolla de manera secuencial. En consecuencia, se espera que con este plan estratégico una organización pueda proyectar el futuro y alcance su visión establecida.

Finalmente, uno de los grandes desafíos en este sector es lograr la fidelidad de los clientes a través de una constante sintonía para satisfacer cada una de sus necesidades y aspiraciones. Es aquí donde se centra gran parte de los esfuerzos para atraer y retener a clientes redituables para las organizaciones. 